\title{
HYDRODYNAMIC COLLIMATION OF YSO JETS
}

\author{
ADAM FRANK \\ Department of Physics and Astronomy, \\ Bausch and Lomb Building, University of Rochester, \\ Rochester, NY 14627-0171, USA \\ AND \\ GARRELT MELLEMA \\ Stockholm Observatory, S-13336 Saltsjöbaden, Sweden
}

\begin{abstract}
We present the results of numerical hydrodynamic models for the collimation of outflows from young stellar objects. We show that the presence of a toroidal environment can lead to efficient formation of jets and bipolar outflows from initially uncollimated central winds. The interaction between the wind and the environment leads to two types of collimation, one which is dominated by radiative cooling effects, and one which works when cooling is less efficient. We describe the two types of jets as they appear in the simulations and we suggest a description for the long term evolution of these structures in more realistic time-dependent wind sources.
\end{abstract}

\section{Introduction}

The physical processes behind the origin of the subjects of this conference, $\mathrm{HH}$ jets, clearly still requires a lot of study. Observations are, however, taking us closer to the young stars and it seems well established that for low mass stars the only way to generate the observed momentum and energy in the jets and outflows is to have some form of MHD process accelerate material into a wind. Beyond this realization it is still not clear what type of MHD process is at work: is it the interaction of a stellar magnetosphere with the inner edge of the accretion disk (the so-called X-wind model, Shu et al. 1994); or a centrifugally driven wind coming off the inner parts of the accretion disk (Königl, 1989; Pudritz, 1991)? It should also be realized that for massive stars the situation is quite different; there the driving 
force behind the wind may be the stellar radiation. It is also not clear if all MHD models can produce well collimated jets on the observed lengthscales, something which was also recently pointed out in a numerical study by Romanova et al. (1996).

Based on these concerns we propose to separate the issues of wind acceleration and jet and bipolar outflow collimation. We believe such a distinction is useful as it allows us to address issues such as the relation between jets and outflows in a focused way and allows the possibility that the acceleration processes may be different in low and high mass stars.

In this work we study the collimating properties of the environment. Our approach is related to the earlier work on hydrodynamic collimation, such as DeLaval nozzles (Königl, 1982; Raga \& Cantó, 1989). Such models have fallen out of favor because of length scale requirements (Königl \& Ruden, 1993) and stability considerations (Koo \& McKee, 1992). We have found however that these objections do not hold when considering the timedependent evolution of these types of flow.

The background to our present study is the high degree of collimation found when studying the interaction of a stellar wind with a toroidal environment in the context of the formation of aspherical Planetary Nebulae (PNe) (Icke et al., 1992). The mechanism has been called "Shock-Focused Inertial Confinement" (SFIC). In the SFIC mechanism it is the inertia of a toroidal environment rather than its thermal pressure, which produces a bipolar wind-blown bubble. In our studies of bipolar PNe formation we found the bubble's wind shock (which decelerates the wind) takes on an aspherical, prolate geometry (Eichler, 1982; Icke et al., 1992). The radially streaming central wind strikes this prolate shock obliquely focusing it towards the polar axis and initiating jet collimation. Other effects such as instabilities along the walls of the bubble, help to maintain the collimation of the shocked wind flow.

This mechanism was first applied to the case of young stars by Frank \& Noriega-Crespo (1994), and more recently by Frank \& Mellema (1996) (henceforth Paper 1) and Mellema \& Frank (1997) (henceforth Paper 2). A related study is the one by Peter \& Eichler (1995).

\section{Numerical Method}

To study the collimating effects of the environment we choose the case where the central wind is maximally uncollimated, i.e. perfectly spherical. This can be considered the worst case scenario for MHD winds since most mechanisms will results in some degree of focusing towards the axis. The central protostellar wind is fixed in an inner sphere of cells on the computational grid. The relevant input parameters are simply the mass loss rate 


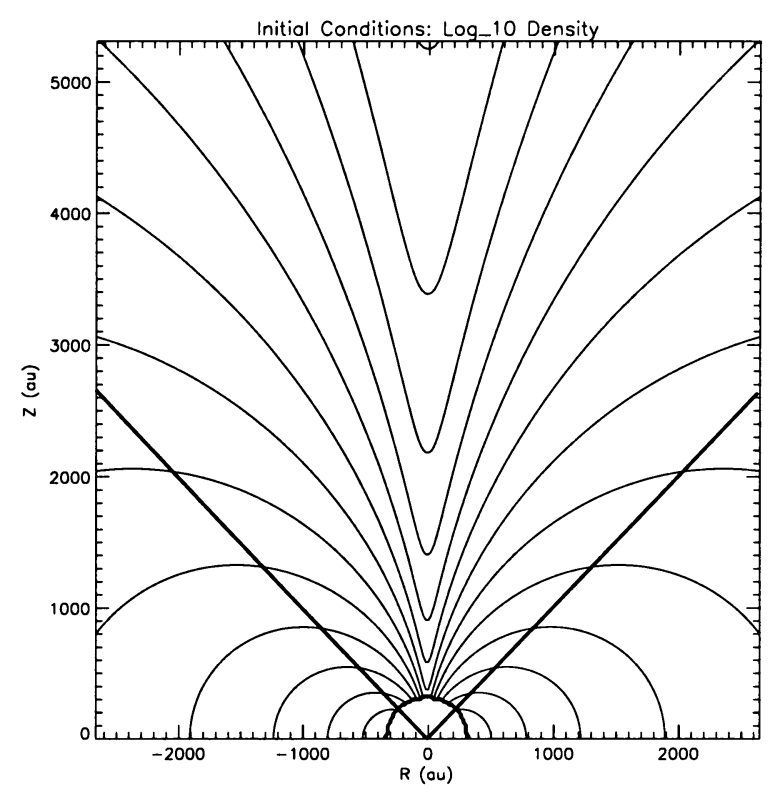

Figure 1. Initial density distribution. Shown are the $\log _{10}$ contours of density from Eq. 1 with an equator to pole contrast $q=70$. The two solid lines show the angle at which $\rho=.5 \rho_{\max }=.5 \rho\left(90^{\circ}\right)$. These occur at $\theta \sim 45^{\circ}$ making the opening angle of the density distribution $\sim 90^{\circ}$

$\dot{M}_{\mathrm{w}}$ and velocity $V_{\mathrm{w}}$ in the wind.

For the environment we choose a density distribution with a toroidal shape. Such toroidal density distributions are theoretically expected for the collapse of a rotating cloud (Terebey, Shu \& Cassen, 1984), a flattened filament (Hartmann, Calvet, \& Boss, 1996), or a magnetized cloud (Li \& Shu, 1996). These are admittedly all axi-symmetric models, and it would be interesting to see how this holds under more general conditions. Lucas \& Roche (1997) present some observational evidence for toroidal environments of proto-stars.

The actual form of the toroid we use is given by

$$
\rho(R, \theta)=\frac{\dot{M}_{a}}{4 \pi R^{2}}\left(\frac{2 G M}{R}\right)^{-\frac{1}{2}}\left\{1-\frac{\zeta}{6}\left[13 P_{2}(\cos (\theta))-1\right]\right\}
$$

in which $R$ is the spherical radius (we will use $r$ to denote the cylindrical radius). In Eq. $1 \dot{M}_{\mathrm{a}}$ is the accretion mass loss rate and $\mathrm{M}$ is the mass of the star. Equation 1 is a modified form of Eq. 96 from Terebey, Shu \& Cassen (1984) (originally derived by Ulrich 1976). We use it here because it produces the required toroidal geometry as well having the $R^{-\frac{3}{2}}$ radial 
dependence, appropriate to a freely falling envelope. The parameter $\zeta$ determines the flattening of the cloud, which we prefer to quantify with the density contrast $q$, the ratio between the density at the equator and at the pole. We note that this density distribution is actually very similar to the solution found by Li \& Shu (1996) for a magnetized cloud. The shape of this density distribution is shown in Fig. 1, which serves to illustrate that the opening angle of the toroid is actually quite wide. A more detailed description of the initial conditions can be found in Paper 1.

The evolution of this system is followed by solving numerically for the well-known Euler equations, including a source term in the energy equation to account for radiative losses. The numerical method used to solve these equations is based on the Total Variation Diminishing (TVD) method of Harten (1983) as implemented by Ryu et al (1995). For cooling we used the standard coronal cooling curve of Dalgarno \& McCray (1972). Details of the methods and the implementation of cooling can be found in Paper 2.

\section{Results without cooling}

In Paper 1 we presented the results of simulations without cooling. Although this is not a realistic assumption in most cases, we considered it to be a good first step. The fact that some of the features of the non-cooling jets also appear in the simulations with cooling (see below), justifies this approach.

Figure 2 shows a typical result from Paper 1. The outflow and environment parameters are: $\dot{M}_{\mathrm{w}}=10^{-7} \mathrm{M}_{\odot} \mathrm{yr}^{-1}, V_{w}=200 \mathrm{~km} \mathrm{~s}^{-1}$, $\dot{M}_{\mathrm{a}}=10^{-5} \mathrm{M}_{\odot} \mathrm{yr}^{-1}, q=70$.

The collimated flow that forms has all the usual features expected for gaseous jets: bow and jet shocks; turbulent cocoons; crossing shocks and internal Mach disks. In Paper 1 we demonstrated that the flow pattern has characteristics of both a supersonic jet and a wind-blown bubble, something which may be important for understanding the connection between jets and molecular outflows. These simulations also showed that strong collimation is principally achieved by the refraction of flow vectors across the aspherical wind shock. Even a small degree of asphericity in the wind shock (a ratio between equatorial and polar shock positions smaller than 0.8 ) is sufficient to produce strong flow focusing (cf. Icke 1988). In the case of stronger asphericities it is even possible to have the flow remain supersonic across the shock.

\section{Results with cooling}

In Paper 2 we presented the results of simulations with radiative cooling included. Although the cooling is only implemented in a simplified way (by 

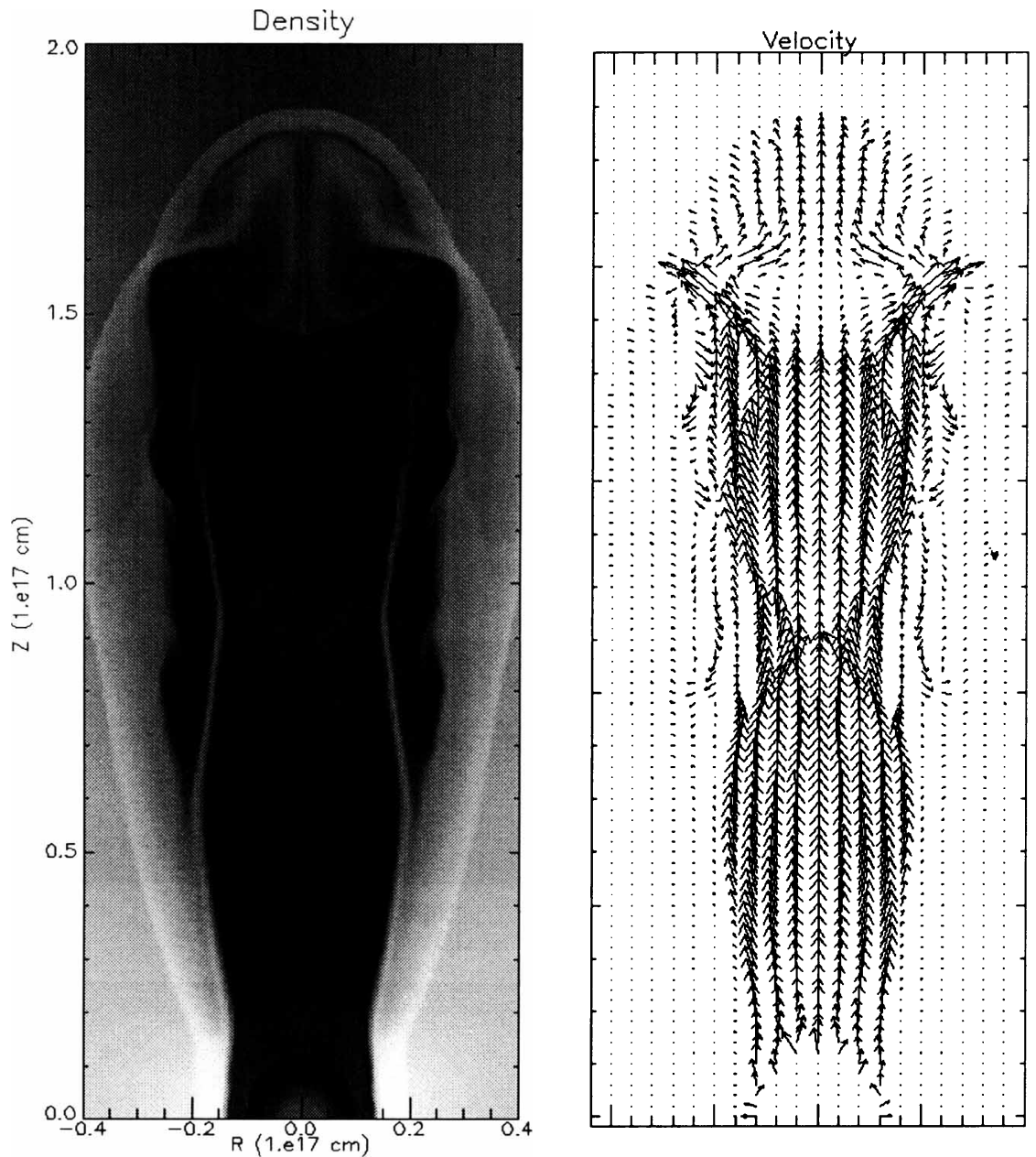

Figure 2. Density and velocity for a non-cooling model. Shown are a gray scale map of $\log _{10}(\rho)$ and a map of velocity vector field for model A after 1035 years of evolution. In the density map dark (light) shades correspond to low (high) densities. In the velocity field map vectors in the inner, freely expanding wind zone have not been plotted. Thus the first "shell" of vectors maps out the wind shock.

using a coronal cooling curve) this should still recover the most important dynamical effects of the cooling.

A typical sequence can be seen in Figs. 3 and 4. In this simulation the outflow has a velocity of $350 \mathrm{~km} \mathrm{~s}^{-1}$ and a mass loss rate of $10^{-7} \mathrm{M}_{\odot} \mathrm{yr}^{-1}$. The environment has a pole to equator contrast of 70 , and an accretion 

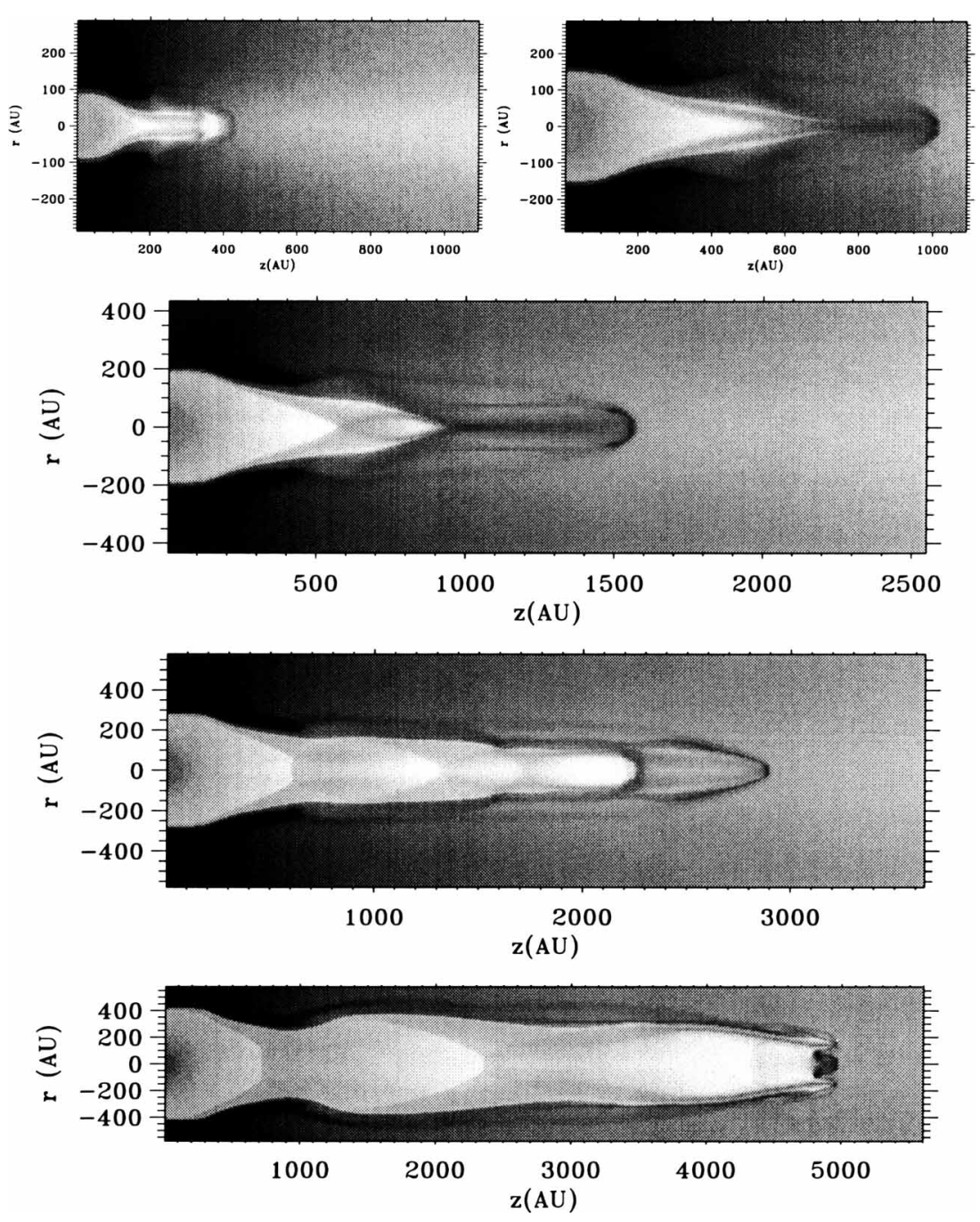

Figure 3. Density evolution for a cooling simulation. The grey scales show $\log _{10}$ contours for times $t=25,62,87,137,225$ years. The darker shades are higher density. One sees the initial radiative phase, the development of the 'cool jet' and the development of the 'hot jet'.

rate $\dot{M}_{\mathrm{a}}=10^{-6} \mathrm{M}_{\odot} \mathrm{yr}^{-1}$. We find that the evolution of the flow proceeds in two phases. First a dense cool jet forms $(t=62$ years $)$, followed at later times by a lower density, hot jet $(t>100$ years). This second 'hot jet' is similar to the jets found in the non-cooling calculations of Paper 1. The sequence is most clearly seen in the temperature plots in Fig. 4.

The formation of this 'cool jet' early in the evolution is an interest- 

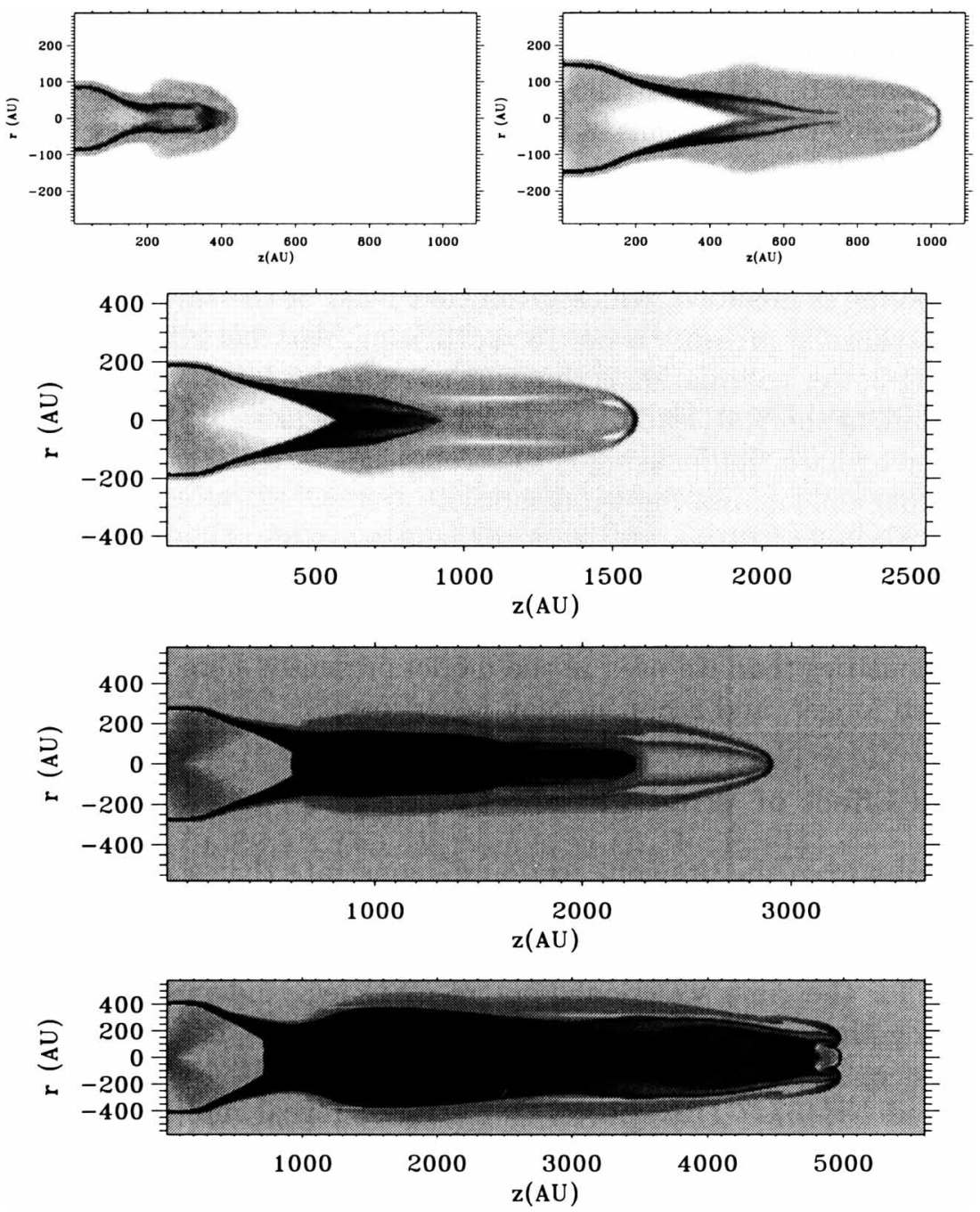

Figure 4. Temperature evolution for a cooling simulation. The grey scales show $\log _{10}$ contours for times $t=25,62,87,137,225$ years. The darker shades are higher temperature. One sees the initial radiative phase, the development of the 'cool jet' and the development of the 'hot jet'. Notice the shape of the inner shock.

ing new effect seen in these radiative simulations. It forms when the bubble is still mostly radiative and its formation mechanism appears to be the same as that of the "converging conical flows" originally proposed by Cantó \& Rodriguez (1980), and later studied analytically and numerically by Cantó, Tenorio-Tagle \& Różyczka (1988) and Tenorio-Tagle, Cantó \& Różyczka (1988). When the thermal energy produced at the wind shock 
is completely lost to radiation the post-shock flow collapses and the only path available for the shocked wind is to slide along the contact discontinuity towards the poles of the bubble, leading to converging conical flows. The collision of fluid parcels at the poles produces a shock wave where the transverse velocity components are lost and a narrow well collimated beam or jet of dense, cool gas is formed.

In general one can say that hydrodynamic collimation is more effective when cooling is included, which given that most of the sideward expansion is due to thermal pressure is not too surprising. Another effect that helps in achieving better collimation is that due to radiative losses the wind shock is much more aspherical, leading to very efficient focusing. In fact even during the hot jet phase the focusing is so strong that at some positions the flow stays supersonic across the wind shock.

The duration of the cool jet phase depends very much on the efficiency of the cooling, and hence on the velocity of the outflow and the density of the wind and the environment. In deeply embedded objects which have higher densities than we used in the model presented here, the cool jet will last much longer, and a hot jet may never form.

\section{The Effect of Wind Variability}

The results from the previous section demonstrate that strong collimation can be achieved from a purely hydrodynamic interaction between winds and protostellar environments. But to apply this model to young stars we must account for the time scales inherent to YSO jets and molecular outflows (assuming that jets are connected to the outflows, Chernin et al. 1994). Recent deep exposure images of HH jets such as HH34 (Bally \& Devine, 1994) and HH46/47 (Heathcote et al., 1996) reveal multiple bow shocks that imply jet lifetimes of many thousands of years or more. In addition, the molecular outflows have dynamical lifetimes on the order of $10^{3}$ to $10^{5}$ years (Bachiller, 1996).

Because our bubbles are not in pressure equilibrium with the environment, they will continue to grow in the lateral direction, leading to wide jets at later times. In order to account for the observed jet widths, this expansion has to be restricted. An attractive means for stopping this equatorial growth comes directly from the observations. As was mentioned above many $\mathrm{HH}$ jets show clear signs of variations in velocity along the jet beams. In the most extreme case the jet appears to be temporarily shut off, which explains the multiple bow-shock structures. In the HH34 superjet Bally \& Devine (1994) find a periodicity in the beam with a period between 300 and 900 years. In addition smaller scale variations with periods $\tau<100$ years are seen in many $\mathrm{HH}$ jet beams (Morse, private communication). It 
is reasonable that the velocity variations in the jet beam are a measure of velocity variations in the source material of the jet. There might also be links with FU Orionis outbursts or related events, which occur on similar time scales as the ones mentioned above (see Bell \& Chick, this volume)

One can therefore envisage a scenario where the wind-blown bubble is driven by a time-variable wind. In our simulations we did not include the effect of either gravity or the inward directed accretion flow. Both of these effects will decelerate the flow and constrain the bubble, particularly near the equator where the bubble radius is small so that the accretion velocity $\left(\propto R^{-0.5}\right)$ and gravitational force density is high.

We developed a simple model for the interaction of a periodic stellar wind with an accreting environment. The model assumes spherical symmetry and strong radiative losses from the wind and ambient shocks so that we can use a thin shell approximation. The equations for mass and momentum conservation for a shell of mass $M_{\mathrm{s}}$ and radius $R_{\mathrm{s}}$ are

$$
\begin{gathered}
\frac{d R_{\mathrm{s}}}{d t}=V_{\mathrm{s}} \\
\frac{d M_{\mathrm{s}}}{d t}=4 \pi R_{\mathrm{s}}{ }^{2} \rho_{\mathrm{w}}\left(R_{\mathrm{s}}\right)\left(V_{\mathrm{w}}-V_{\mathrm{s}}\right)+4 \pi R_{\mathrm{s}}{ }^{2} \rho_{\mathrm{a}}\left(R_{\mathrm{s}}\right)\left(V_{\mathrm{s}}+V_{\mathrm{a}}\right) \\
\frac{d M_{\mathrm{s}} V_{\mathrm{s}}}{d t}=4 \pi R_{\mathrm{s}}{ }^{2} \rho_{\mathrm{w}}\left(R_{\mathrm{s}}\right) V_{\mathrm{w}}\left(V_{\mathrm{w}}-V_{\mathrm{s}}\right)-4 \pi R_{\mathrm{s}}{ }^{2} \rho_{\mathrm{a}}\left(R_{\mathrm{s}}\right) V_{\mathrm{a}}\left(V_{\mathrm{s}}+V_{\mathrm{a}}\right)-\frac{G M_{*} M_{\mathrm{s}}}{R_{\mathrm{s}}{ }^{2}}
\end{gathered}
$$

(Volk \& Kwok, 1985). Here $\rho_{a}$ and $V_{a}$ are the radially dependent accretion flow density and velocity. These equations can be rewritten in the form of a simple set of coupled ODEs. We solved the ODEs using a 4th order Runge-Kutta method with an adaptive step-size. For the variable wind we used a sinusoidal variation with an amplitude of $100 . \dot{M}_{\mathrm{w}}$ is kept constant, but we have also tried the case in which $\dot{M}_{\mathrm{w}}$ varies in the same way as $V_{\mathrm{w}}$, which gives similar results.

The parameters for the wind and the environment are the same as for the model shown in Sect. 4. Other details of the model can be found in Paper 2. We assume that before the start of the integration, the bubble was set in motion, perhaps by an energetic episodic outburst from the protostar. This initial condition gives a total energy in the shell of $10^{40}$ ergs which is more than five orders of magnitude less than what is released in a typical FU Orionis outburst (Hartmann \& Kenyon, 1996).

The results for variable winds with periods of $\tau_{\mathrm{w}}=10^{6}, 500,400,300$ and 200 years are shown in Fig. 5. For the constant wind $\left(\tau_{\mathrm{w}}=10^{6}\right.$ years $)$ the bubble expands monotonically although it does experience changes in velocity due to accretion ram pressure and gravitational forces. When the wind is allowed to vary, these forces produce dramatic changes in the bubble's evolution. For all four variation periods we see that the expansion 


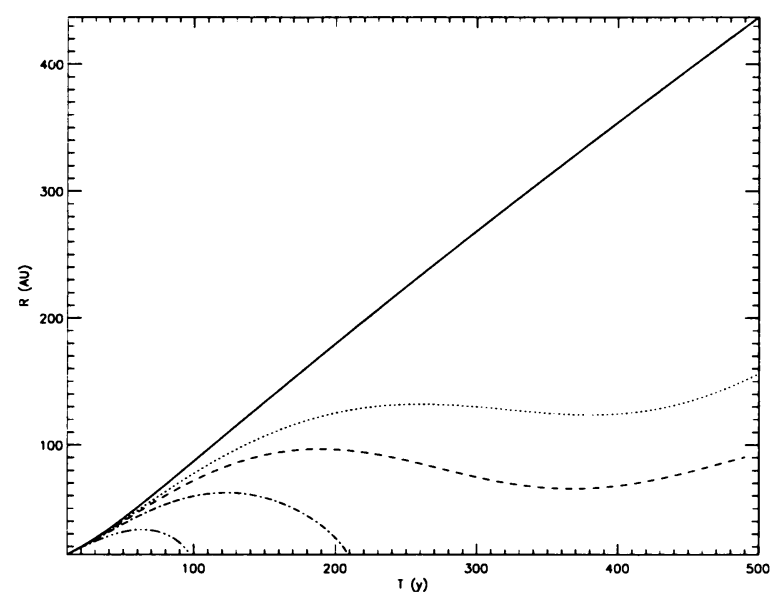

Figure 5. Evolution of spherical wind blown bubbles in accretion environment. Shown are the radius of 5 bubbles driven winds with different periods as a function of time. Solid line corresponds to a period of $P=10^{6}$ years. Dotted line: $P=500$ years. Dashed line: $P=400$ years. Dashed-dot line: $P=300$ years. Dashed-dot-dot line: $P=200$ years.

of the bubble can be reversed $\left(V_{\mathrm{s}}<0\right)$ for some time. For longer periods the bubble gains enough momentum before the wind enters a minimum to either continue a slow expansion $\left(\tau_{\mathrm{w}}=500\right.$ years) or maintain a constant average radius ( $\tau_{\mathrm{w}}=400$ years). For shorter periods the bubble is "crushed" during the wind's quiescent phase. Note that we ended the calculation if the shock radius $R_{\mathrm{s}}$ became smaller than $10^{12} \mathrm{~cm}$.

From these results we conclude that, in principle, time varying winds can produce wind-blown bubbles whose size does not increase beyond some upper limit. If these bubbles produce jets through the hydrodynamic mechanisms described above, the model age and collimation scales can be made consistent with observations. We imagine that during a periodic increase in mechanical luminosity the YSO will begin driving a bubble which in turn produces collimated jets. As the wind speed varies the bubble either oscillates around some average radius (producing variations in the jet beam) or it collapses entirely. Jet production begins again when the momentum in the wind has increased enough to produce another bubble.

\section{Connection to observations}

The results so far can be considered as physical experiments rather than models for specific observed objects, since there are several important effects which we have left out of the numerical simulations (gravity, accretion flow, 
rotation). Clearly the next step should be to include these, and also to add microphysics and diagnostics to make predictions of observables, similarly to the work of Frank \& Mellema (1994) and Suttner et al (1997). This will allow us to check whether the cool jets reproduce the observables in terms of temperature and density and in how far the hot jet can be observed.

There are however already two observational results in support of the model. Firstly the detection of non-thermal radio emission from the source W3(OH) (Reid et al., 1995). These authors found strong synchrotron emission arising at the center of a linear chain of maser sources. Recent high resolution images show the synchrotron emission actually appears as a two sided jet emanating from the geometric center of the two maser flows (Wilner et $a l, 1997)$. Such a morphology is likely to occur from first-order Fermi acceleration at strong shocks around the central object.

Secondly a geometry very reminiscent of the converging conical flows has been observed in the proto-planetary nebulae He 3-1475 (Harrington, private communication), an object which is producing collimated outflows with velocities of several times $100 \mathrm{~km} \mathrm{~s}^{-1}$ (Riera et al., 1995).

\section{Conclusions}

- The interaction of an outflow with a toroidal environment is an efficient means to collimate unfocused or wide-angle winds.

- The interaction gives rise to two jet phases, an initial 'cool jet', which form due to converging conical flows at the top of the bubble, and a later 'hot jet' which forms when cooling starts to be less efficient. The cool jet is dense and is the one which should be most easily observed.

- Both the variability and the longevity of the jets could be explained by a model in which the outflow is time-dependent, producing a series of cool jets.

- The occurrence of non-thermal emission in some radio jets can be seen as supporting our model, since its production requires strong shocks.

Acknowledgments: The authors gratefully acknowledge Dong-Su Ryu for making the TVD code available to us. We also benefited from discussions with Alberto Noriega-Crespo, Alex Raga, Mark Reid, Tom Jones. This work was supported by the Minnesota Supercomputer Institute.

\section{References}

Bachiller, R., 1996, ARAA, 34, 111

Bally, J., Devine, D., 1994, ApJ, 428, L65

Cantó, J., Rodrí guez, L.F. 1980, ApJ, 239, 982

Cantó, J., Tenorio-Tagle, G., Różyczka, M. 1988, A\&A, 192, 287

Chernin, L.M. Masson, C.R., Dal Pino, E.M., Benz, W., 1994, ApJ, 426, 204 
Dalgarno, A., McCray, R., 1972, ARAA, 10, 375

Eichler, D., 1982, ApJ, 263, 571

Frank, A., Noriega-Crespo, A., 1994, A\&A, 290, 643

Frank, A., Mellema, G., 1994, ApJ, 430800

Frank, A., Mellema, G., 1996, ApJ, 472, 684 (Paper 1)

Harten, A., 1983, J. Comp. Phys, 49357

Hartmann, L., Calvet, N., Boss, A., 1996, ApJ, 464, 387

Hartmann, L., Kenyon, S., 1996, ARAA, 34, 207

Heathcote, S., Morse, J., Hartigan, P., Reipurth, B., Schwartz, R., Bally, J., Stone, J., 1996, AJ 112, 1141

Henriksen, R., Ptuskin, V., Mirabel I., 1991 A\&A, 248, 221

Icke, V., 1988, A\&A, 202, 177

Icke, V., Balick, B., Frank, A., 1992, A\&A, 253, 224

Ip, W.H., 1995, A\&A, 300, 283

Königl, A., 1982 ApJ, 261, 115

Königl, A., 1989 ApJ, 342, 208

Königl, A., Ruden, S.P. 1993, in "Protostars and Planets III", E.H. Levy, J.I. Lunine (eds). Univ. of Arizona Press. p. 641

Koo, B., McKee, C.F., 1992, ApJ, 388, 103

Li, Z., \& Shu, F., 1996, ApJ, 472, 211

Lucas, P.W., Roche, P.F., 1997, MNRAS in press

Mellema, G., Eulderink, F., Icke, V., 1991, A\&A, 252, 718

Mellema, G., Frank, A., 1997, submitted to MNRAS (Paper 2)

Peter, W. Eichler, D, 1995, ApJ, 438, 244

Pudritz, R.E., 1991, in "The Physics of Star Formation and Early Stellar Evolution", C.J. Lada and N.D. Kylafis (eds). NATO ASI Series, Kluwer, Dordrecht. p. 365

Raga, A.C., Cantó, J., 1989 ApJ, 344404

Reid, M. J., Argon, A. L., Masson, C. R., Menten, K. M. Moran, J. M., 1995, ApJ, 443, 238

Riera, A., Garcia-Laro, P., Manchado, A., Pottasch, S.R., Raga, A.C., 1995, A\&A, 302, 137

Romanova, M., Ustyugova, G., Koldoba, A., Chechetkin, V., Lovelace, R., 1996, preprint Ryu, D., Brown, G.L., Ostriker, J.P., Loeb, A., ApJ, 452, 364

Shu, F., Najiata, J., Ostriker, E., Wilkin, F., Ruden, S., Lizano, S., 1994, ApJ, 429, 781

Suttner, G., Smith, M.D., Yorke, H.W., Zinnecker, H., 1997, A\&A, 318, 595

Tenorio-Tagle, G., Cantó, J., Różyczka, M., 1988, A\&A, 202, 256

Terebey, S., Shu, F., Cassen, P., 1984, ApJ, 286, 529

Ulrich, R.K., 1976, ApJ, 210, 377

Volk, K., Kwok, S., 1985, A\&A, 153, 79

Wilner, D.J., Reid, M.J., Menten, K.M., Moran, J.M, 1997, in: Poster proceedings of IAU Symposium 182 on Herbig-Haro Objects and the Birth of Low Mass Stars, F. Malbet, A. Castets (eds.). Obs. de Grenoble. p. 193 